\title{
A modified subgradient extragradient method for solving monotone variational inequalities
}

\author{
Songnian $\mathrm{He}^{1,2^{*}}$ and Tao $\mathrm{Wu}^{1}$
}

\author{
"Correspondence: \\ songnianhe@163.com \\ 'College of Science, Civil Aviation \\ University of China, Tianjin, 300300, \\ China \\ ${ }^{2}$ Tianjin Key Laboratory for \\ Advanced Signal Processing, Civil \\ Aviation University of China, Tianjin \\ 300300, China
}

\begin{abstract}
In the setting of Hilbert space, a modified subgradient extragradient method is proposed for solving Lipschitz-continuous and monotone variational inequalities defined on a level set of a convex function. Our iterative process is relaxed and self-adaptive, that is, in each iteration, calculating two metric projections onto some half-spaces containing the domain is involved only and the step size can be selected in some adaptive ways. A weak convergence theorem for our algorithm is proved. We also prove that our method has $O\left(\frac{1}{n}\right)$ convergence rate.
\end{abstract}

MSC: 47J20; 90C25; 90C30; 90C52

Keywords: variational inequalities; subgradient extragradient method; Lipschitz-continuous mapping; level set; half-spaces; convergence rate

\section{Introduction}

Let $H$ be a real Hilbert space with inner product $\langle\cdot, \cdot\rangle$ and norm $\|\cdot\|$. The variational inequality problem $(V I P)$ is aimed to finding a point $x^{*} \in C$, such that

$$
\left\langle f\left(x^{*}\right), x-x^{*}\right\rangle \geq 0, \quad \forall x \in C,
$$

where $C$ is a nonempty closed convex subset of $H$ and $f: C \rightarrow H$ is a given mapping. This problem and its solution set are denoted by $\operatorname{VI}(C, f)$ and $\operatorname{SOL}(C, f)$, respectively. We also always assume that $\operatorname{SOL}(C, f) \neq \emptyset$. The variational inequality problem $\operatorname{VI}(C, f)$ has received much attention due to its applications in a large variety of problems arising in structural analysis, economics, optimization, operations research and engineering sciences; see [1-14] and the references therein.

It is well known that the problem (1.1) is equivalent to the fixed point problem for finding a point $x^{*} \in C$, such that [1]

$$
x^{*}=P_{C}\left(x^{*}-\lambda f\left(x^{*}\right)\right)
$$

where $\lambda$ is an arbitrary positive constant. Many algorithms for the problem (1.1) are based on the fixed point problem (1.2). Korpelevich [2] proposed an algorithm for solving the

(c) The Author(s) 2017. This article is distributed under the terms of the Creative Commons Attribution 4.0 International License (http://creativecommons.org/licenses/by/4.0/), which permits unrestricted use, distribution, and reproduction in any medium, provided you give appropriate credit to the original author(s) and the source, provide a link to the Creative Commons license, and indicate if changes were made. 
problem (1.1) in Euclidean space $R^{n}$, known as the extragradient method (EG):

$$
\begin{aligned}
& x_{0} \in C, \\
& \tilde{x}_{n}=P_{C}\left(x_{n}-\lambda f\left(x_{n}\right)\right), \\
& x_{n+1}=P_{C}\left(x_{n}-\lambda f\left(\tilde{x}_{n}\right)\right),
\end{aligned}
$$

where $\lambda$ is some positive number and $P_{C}$ denotes the metric projection of $H$ onto $C$. She proved that if $f$ is $\kappa$-Lipschitz-continuous and $\lambda$ is selected such that $\lambda \in(0,1 / \kappa)$, then the two sequences $\left\{x_{n}\right\}$ and $\left\{\tilde{x}_{n}\right\}$ generated by the EG method, converge to the same point $z \in \operatorname{SOL}(C, f)$.

In 2006, Nadezhkina and Takahashi [3] generalized the above EG method to general Hilbert spaces (including infinite-dimensional spaces) and they also established the weak convergence theorem.

In each iteration of the EG method, in order to get the next iterate $x_{k+1}$, two projections onto $C$ need to be calculated. But projections onto a general closed and convex subset are not easily executed and this might greatly affect the efficiency of the EG method. In order to overcome this weakness, Censor et al. developed the subgradient extragradient method in Euclidean space [4], in which the second projection in (1.4) onto $C$ was replaced with a projection onto a specific constructible half-space, actually which is one of the subgradient half-spaces. Then, in [5, 6], Censor et al. studied the subgradient extragradient method for solving the VIP in Hilbert spaces. They also proved the weak convergence theorem under the assumption that $f$ is a Lipschitzian continuous and monotone mapping.

The main purpose of this paper is to propose an improved subgradient extragradient method for solving the Lipschitz-continuous and monotone variational inequalities defined on a level set of a convex function [13], that is, $C:=\{x \in H \mid c(x) \leq 0\}$ and $c: H \rightarrow R$ is a convex function. In our algorithm, two projections $P_{C}$ in (1.3) and (1.4) will be replaced with $P_{C_{k}}$ and $P_{T_{k}}$, respectively, where $C_{k}$ and $T_{k}$ are half-spaces, such that $C_{k} \supset C$ and $T_{k} \supset C . C_{k}$ is based on the subdifferential inequality, the idea of which was proposed firstly by Fukushima [14], and $T_{k}$ is the same one as Censor's method [5].

It is also worth pointing out that the step size in our algorithm can be selected in some adaptive way, that is, we have no need to know or to estimate any information as regards the Lipschitz constant of $f$, therefore, our algorithm is easily executed.

Our paper is organized as follows. In Section 2, we list some basic definitions, properties and lemmas. In Section 3, the improved subgradient extragradient algorithm and its corresponding geometrical intuition are presented. In Section 4, the weak convergence theorem for our method is proved. Finally, we prove that our algorithm has $O\left(\frac{1}{n}\right)$ convergence rate in the last section.

\section{Preliminaries}

In this section, we list some basic concepts and lemmas, which are useful for constructing the algorithm and analyzing the convergence. Let $H$ be a real Hilbert space with inner product $\langle\cdot, \cdot\rangle$ and norm $\|\cdot\|$ and let $C$ be a closed convex subset of $H$. We write $x_{k} \rightarrow$ $x$ and $x_{k} \rightarrow x$ to indicate that the sequence $\left\{x_{k}\right\}_{k=0}^{\infty}$ converges weakly and strongly to $x$, respectively. For each point $x \in H$, there exists a unique nearest point in $C$, denoted by 
$P_{C}(x)$, such that

$$
\left\|x-P_{C}(x)\right\| \leq\|x-y\|, \quad \forall y \in C .
$$

The mapping $P_{C}: H \rightarrow C$ is called the metric projection of $H$ onto $C$. It is well known that $P_{C}$ is characterized by the following inequalities:

$$
\begin{aligned}
& \left\langle x-P_{C}(x), P_{C}(x)-y\right\rangle \geq 0, \\
& \|x-y\|^{2} \geq\left\|x-P_{C}(x)\right\|^{2}+\left\|y-P_{C}(x)\right\|^{2},
\end{aligned}
$$

for all $x \in H, y \in C[15,16]$.

A function $c: H \rightarrow R$ is said to be Gâteaux differentiable at $x \in H$, if there exists an element, denoted by $c^{\prime}(x) \in H$, such that

$$
\lim _{t \rightarrow 0} \frac{c(x+t v)-c(x)}{t}=\left\langle v, c^{\prime}(x)\right\rangle, \quad \forall v \in H,
$$

where $c^{\prime}(x)$ is called the Gâteaux differential of $c$ at $x$. We say $c$ is Gâteaux differentiable on $H$, if for each $x \in H, c$ is Gâteaux differentiable at $x$.

A function $c: H \rightarrow R$ is said to be weakly lower semicontinuous (w-lsc) at $x \in H$, if $x_{k} \rightarrow x$ implies $c(x) \leq \liminf _{k \rightarrow \infty} c\left(x_{k}\right)$. We say $c$ is weakly lower semicontinuous on $H$, if for each $x \in H, c$ is weakly lower semicontinuous at $x$.

A function $c: H \rightarrow R$ is called convex, if we have the inequality

$$
c(t x+(1-t) y) \leq t c(x)+(1-t) c(y)
$$

for all $t \in[0,1]$ and $x, y \in H$.

For a convex function $c: H \rightarrow R, c$ is said to be subdifferentiable at a point $x \in H$ if the set

$$
\partial c(x) \triangleq\{d \in H \mid c(y) \geq c(x)+\langle d, y-x\rangle, \forall y \in H\}
$$

is not empty, where each element in $\partial c(x)$ is called a subgradient of $c$ at $x, \partial c(x)$ is subdifferential of $c$ at $x$ and the inequality in (2.4) is said to be the subdifferential inequality of $c$ at $x$. We say $c$ is subdifferentiable on $H$, if $c$ is subdifferentiable at each $x \in H$. It is well known that if $c$ is Gâteaux differentiable at $x$, then $c$ is subdifferentiable at $x$ and $\partial c(x)=\left\{c^{\prime}(x)\right\}$, namely, $\partial c(x)$ is just a set of the simple points [17].

A mapping $f: H \rightarrow H$ is said to be Lipschitz-continuous [15], if there exists a positive constant $\kappa$, such that

$$
\|f(x)-f(y)\| \leq \kappa\|x-y\|, \quad \forall x, y \in H .
$$

$f$ is also said to be a $\kappa$-Lipschitzian-continuous mapping.

A mapping $f: H \rightarrow H$ is said to be monotone on $H$, if

$$
\langle f(x)-f(y), x-y\rangle \geq 0, \quad \forall x, y \in H .
$$


Definition 2.1 (Normal cone) We denote the normal cone by $N_{C}(v)[18]$ of $C$ at $v \in C$, i.e.

$$
N_{C}(v):=\{w \in H \mid\langle w, y-v\rangle \leq 0, \forall y \in C\} .
$$

Definition 2.2 (Maximal monotone operator) Let $T: H \rightrightarrows 2^{H}$ be a point-to-set operator defined on $H$. $T$ is called a maximal monotone operator if $T$ is monotone, i.e.

$$
\langle u-v, x-y\rangle \geq 0, \quad \forall u \in T(x) \text { and } \forall v \in T(y)
$$

and the graph $G(T)$ of $T$,

$$
G(T):=\{(x, u) \in H \times H \mid u \in T(x)\},
$$

is not properly contained in the graph of any other monotone operator.

It is clear that a monotone mapping $T$ is maximal iff for any $(x, u) \in H \times H$, if $\langle u-v, x-$ $y\rangle \geq 0, \forall(y, v) \in G(T)$, then it follows that $u \in T(x)$.

Define

$$
T v= \begin{cases}f(v)+N_{C}(v) & \text { if } v \in C, \\ \emptyset, & \text { if } v \notin C .\end{cases}
$$

Then $T$ is maximal monotone and $0 \in T v$ if and only if $v \in \operatorname{SOL}(C, f)[18]$.

The next property is known as the Opial condition and all Hilbert spaces have this property [19].

Lemma 2.3 For any sequence $\left\{x_{k}\right\}_{k=0}^{\infty}$ in $H$ that converges weakly to $x\left(x_{k} \rightarrow x\right)$, the inequality

$$
\liminf _{n \rightarrow \infty}\left\|x_{k}-x\right\|<\liminf _{n \rightarrow \infty}\left\|x_{k}-y\right\|
$$

holds for any $y \in H$ with $x \neq y$.

The following lemma was proved in [20].

Lemma 2.4 Let $H$ be a real Hilbert space and let $C$ be a nonempty, closed and convex subset of $H$. Let the sequence $\left\{x_{k}\right\}_{k=0}^{\infty} \subset H$ be Fejér-monotone with respect to $C$, i.e., for any $u \in C$,

$$
\left\|x_{k+1}-u\right\| \leq\left\|x_{k}-u\right\|, \quad \forall k \geq 0 .
$$

Then $\left\{P_{C}\left(x_{k}\right)\right\}_{k=0}^{\infty}$ converges strongly to some $z^{\prime} \in C$. 


\section{The modified subgradient extragradient method}

In this section, we give our algorithm for solving the $\operatorname{VI}(C, f)$ in the setting of Hilbert spaces, where $C$ is a level set given as follows:

$$
C:=\{x \in H \mid c(x) \leq 0\}
$$

where $c: H \rightarrow R$ is a convex function.

In the rest of this paper, we always assume that the following conditions are satisfied.

Condition 3.1 The solution set of $\operatorname{VI}(C, f)$, denoted by $\operatorname{SOL}(C, f)$, is nonempty.

Condition 3.2 The mapping $f: H \rightarrow H$ is monotone and Lipschitz-continuous on $H$ (but we have no need to know or to estimate the Lipschitz constant of $f$ ).

Condition 3.3 The function $c: H \rightarrow R$ satisfies the following conditions:

(i) $c(x)$ is a convex function;

(ii) $c(x)$ is weakly lower semicontinuous on $H$;

(iii) $c(x)$ is Gâteaux differentiable on $H$ and $c^{\prime}(x)$ is a $M_{1}$-Lipschitzian-continuous mapping on $H$;

(iv) there exists a positive constant $M_{2}$ such that $\|f(x)\| \leq M_{2}\left\|c^{\prime}(x)\right\|$ for any $x \in \partial C$, where $\partial C$ denotes the boundary of $C$.

Next, we present the modified subgradient extragradient method as follows.

Algorithm 3.4 (The modified subgradient extragradient method)

Step 1: select an initial guess $x_{0} \in H$ arbitrarily, set $k=0$ and construct the half-space

$$
C_{k}:=\left\{w \in H \mid c\left(x_{k}\right)+\left\langle c^{\prime}\left(x_{k}\right), w-x_{k}\right\rangle \leq 0\right\}
$$

Step 2: given the current iteration $x_{k}$, compute

$$
y_{k}=P_{C_{k}}\left(x_{k}-\beta_{k} f\left(x_{k}\right)\right) \text {, }
$$

where

$$
\beta_{k}=\sigma \rho^{m_{k}}, \quad \sigma>0, \rho \in(0,1)
$$

and $m_{k}$ is the smallest nonnegative integer, such that

$$
\beta_{k}^{2}\left\|f\left(x_{k}\right)-f\left(y_{k}\right)\right\|^{2}+2 M \beta_{k}\left\|x_{k}-y_{k}\right\|^{2} \leq v^{2}\left\|x_{k}-y_{k}\right\|^{2},
$$

where $M=M_{1} M_{2}$ and $v \in(0,1)$.

Step 3: calculate the next iterate,

$$
x_{k+1}=P_{T_{k}}\left(x_{k}-\beta_{k} f\left(y_{k}\right)\right) \text {, }
$$




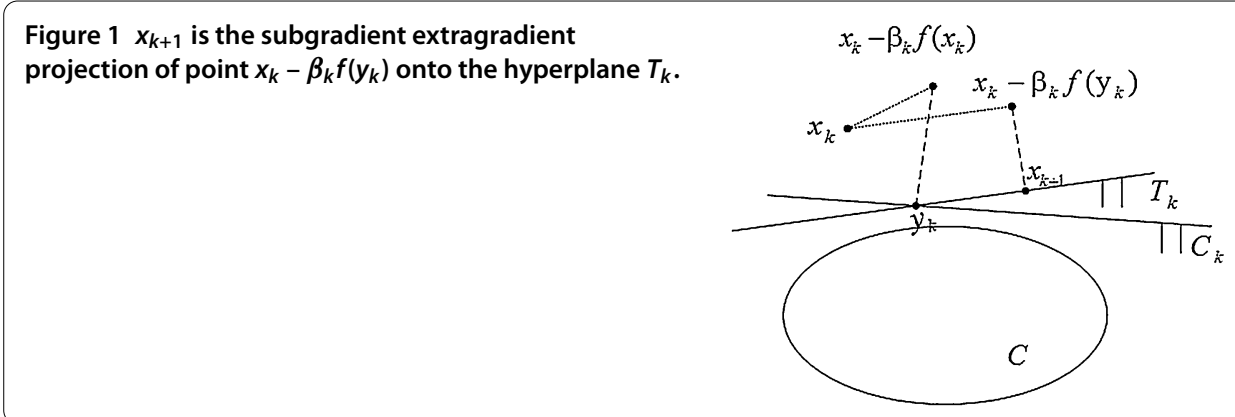

where

$$
T_{k}=\left\{w \in H \mid\left\langle x_{k}-\beta_{k} f\left(x_{k}\right)-y_{k}, w-y_{k}\right\rangle \leq 0\right\},
$$

which is the same half-space as Censor's method [5].

Figure 1 illustrates the iterative steps of this algorithm.

At the end of this section, we list the alternating theorem [21,22] for the solutions of $\operatorname{VI}(C, f)$, where $C$ is given by (3.1). This result will be used to prove the convergence theorem of our algorithm in the next section.

Theorem 3.5 Assume that the solution set $\mathrm{SOL}(C, f)$ of $\mathrm{VI}(C, f)$ is nonempty. Given $x^{*} \in C$. Then $x^{*} \in \operatorname{SOL}(C, f)$ iff we have either

1. $f\left(x^{*}\right)=0$, or

2. $x^{*} \in \partial C$ and there exists a positive constant $\beta$ such that $f\left(x^{*}\right)=-\beta c^{\prime}\left(x^{*}\right)$.

\section{Convergence theorem of the algorithm}

In this section, we prove the weak convergence theorem for Algorithm 3.4. First of all, we give the following lemma, which plays a crucial role in the proof of our main result.

Lemma 4.1 Let $\left\{x_{k}\right\}_{k=0}^{\infty}$ and $\left\{y_{k}\right\}_{k=0}^{\infty}$ be the two sequences generated by Algorithm 3.4. Let $u \in \operatorname{SOL}(C, f)$ and let $\beta_{k}$ be selected as (3.3) and (3.4). Then, under the Conditions 3.1, 3.2 and 3.3 , we have

$$
\left\|x_{k+1}-u\right\|^{2} \leq\left\|x_{k}-u\right\|^{2}-\left(1-v^{2}\right)\left\|x_{k}-y_{k}\right\|^{2}, \quad \forall k \geq 0 .
$$

Proof Taking $u \in \operatorname{SOL}(C, f)$ arbitrarily, for all $k \geq 0$, using (2.3) and the monotonicity of $f$, we have

$$
\begin{aligned}
\left\|x_{k+1}-u\right\|^{2} \leq & \left\|x_{k}-\beta_{k} f\left(y_{k}\right)-u\right\|^{2}-\left\|x_{k}-\beta_{k} f\left(y_{k}\right)-x_{k+1}\right\|^{2} \\
= & \left\|x_{k}-u\right\|^{2}-\left\|x_{k}-x_{k+1}\right\|^{2}+2 \beta_{k}\left\langle f\left(y_{k}\right), u-x_{k+1}\right\rangle \\
= & \left\|x_{k}-u\right\|^{2}-\left\|x_{k}-x_{k+1}\right\|^{2}+2 \beta_{k}\left[\left\langle f\left(y_{k}\right)-f(u), u-y_{k}\right\rangle\right. \\
& \left.+\left\langle f(u), u-y_{k}\right\rangle+\left\langle f\left(y_{k}\right), y_{k}-x_{k+1}\right\rangle\right] \\
\leq & \left\|x_{k}-u\right\|^{2}-\left\|x_{k}-x_{k+1}\right\|^{2} \\
& +2 \beta_{k}\left[\left\langle f(u), u-y_{k}\right\rangle+\left\langle f\left(y_{k}\right), y_{k}-x_{k+1}\right\rangle\right]
\end{aligned}
$$




$$
\begin{aligned}
= & \left\|x_{k}-u\right\|^{2}-\left\|x_{k}-y_{k}\right\|^{2}-\left\|y_{k}-x_{k+1}\right\|^{2}-2\left\langle x_{k}-y_{k}, y_{k}-x_{k+1}\right\rangle \\
& +2 \beta_{k}\left[\left\langle f(u), u-y_{k}\right\rangle+\left\langle f\left(y_{k}\right), y_{k}-x_{k+1}\right\rangle\right] \\
= & \left\|x_{k}-u\right\|^{2}-\left\|x_{k}-y_{k}\right\|^{2}-\left\|y_{k}-x_{k+1}\right\|^{2} \\
& +2\left\langle x_{k}-\beta_{k} f\left(y_{k}\right)-y_{k}, x_{k+1}-y_{k}\right\rangle+2 \beta_{k}\left\langle f(u), u-y_{k}\right\rangle .
\end{aligned}
$$

By the definition of $T_{k}$, we get

$$
\begin{aligned}
\left\langle x_{k}\right. & \left.-\beta_{k} f\left(y_{k}\right)-y_{k}, x_{k+1}-y_{k}\right\rangle \\
& =\left\langle x_{k}-\beta_{k} f\left(x_{k}\right)-y_{k}, x_{k+1}-y_{k}\right\rangle+\beta_{k}\left|f\left(x_{k}\right)-f\left(y_{k}\right), x_{k+1}-y_{k}\right\rangle \\
& \leq \beta_{k}\left|f\left(x_{k}\right)-f\left(y_{k}\right), x_{k+1}-y_{k}\right\rangle \\
& \leq \beta_{k}\left\|f\left(x_{k}\right)-f\left(y_{k}\right)\right\|\left\|x_{k+1}-y_{k}\right\| .
\end{aligned}
$$

Substituting (4.3) into the last inequality of (4.2), thus we obtain

$$
\begin{aligned}
\left\|x_{k+1}-u\right\|^{2} \leq & \left\|x_{k}-u\right\|^{2}-\left\|x_{k}-y_{k}\right\|^{2}-\left\|y_{k}-x_{k+1}\right\|^{2} \\
& +2 \beta_{k}\left\|f\left(x_{k}\right)-f\left(y_{k}\right)\right\|\left\|x_{k+1}-y_{k}\right\| \\
& +2 \beta_{k}\left|f(u), u-y_{k}\right\rangle \\
\leq & \left\|x_{k}-u\right\|^{2}-\left\|x_{k}-y_{k}\right\|^{2}+2 \beta_{k}\left\langle f(u), u-y_{k}\right\rangle \\
& +\beta_{k}^{2}\left\|f\left(x_{k}\right)-f\left(y_{k}\right)\right\|^{2} .
\end{aligned}
$$

The subsequent proof is divided into following two cases.

Case 1: $f(u) \neq 0$.

Using Theorem 3.5, there exists a $\beta_{u}>0$ such that $f(u)=-\beta_{u} c^{\prime}(u)$. By the subdifferential inequality, we have

$$
c(u)+\left\langle c^{\prime}(u), y_{k}-u\right\rangle \leq c\left(y_{k}\right), \quad \forall k \geq 0 .
$$

Noting the fact that $c(u)=0$ due to $u \in \partial C$, we have

$$
\left\langle c^{\prime}(u), y_{k}-u\right\rangle \leq c\left(y_{k}\right), \quad \forall k \geq 0 .
$$

Since $-\beta_{u}<0$, it follows from (4.6) that

$$
\left\langle-\beta_{u} c^{\prime}(u), y_{k}-u\right\rangle \geq-\beta_{u} c\left(y_{k}\right), \quad \forall k \geq 0,
$$

it implies

$$
\left\langle f(u), y_{k}-u\right\rangle \geq-\beta_{u} c\left(y_{k}\right), \quad \forall k \geq 0
$$

or

$$
\left\langle f(u), u-y_{k}\right\rangle \leq \beta_{u} c\left(y_{k}\right), \quad \forall k \geq 0 .
$$


By the definition of $C_{k}$, we have

$$
c\left(x_{k}\right)+\left\langle c^{\prime}\left(x_{k}\right), y_{k}-x_{k}\right\rangle \leq 0, \quad \forall k \geq 0
$$

using the subdifferential inequality again,

$$
c\left(y_{k}\right)+\left\langle c^{\prime}\left(y_{k}\right), x_{k}-y_{k}\right\rangle \leq c\left(x_{k}\right), \quad \forall k \geq 0 .
$$

Adding the above two inequalities, we obtain

$$
c\left(y_{k}\right) \leq\left\langle c^{\prime}\left(y_{k}\right)-c^{\prime}\left(x_{k}\right), y_{k}-x_{k}\right\rangle, \quad \forall k \geq 0
$$

Combining (4.7) and (4.8), we have by using (iii) and (iv) of Condition 3.3

$$
\begin{aligned}
\left\langle f(u), u-y_{k}\right\rangle & \leq \beta_{u} c\left(y_{k}\right) \\
& \leq \beta_{u}\left\langle c^{\prime}\left(y_{k}\right)-c^{\prime}\left(x_{k}\right), y_{k}-x_{k}\right\rangle \\
& \leq M\left\|y_{k}-x_{k}\right\|^{2},
\end{aligned}
$$

where $M$ is defined as before. Substituting (4.9) into the last inequality of (4.4), we obtain

$$
\begin{aligned}
\left\|x_{k+1}-u\right\|^{2} \leq & \left\|x_{k}-u\right\|^{2}-\left\|x_{k}-y_{k}\right\|^{2}+2 M \beta_{k}\left\|y_{k}-x_{k}\right\|^{2} \\
& +\beta_{k}^{2}\left\|f\left(x_{k}\right)-f\left(y_{k}\right)\right\|^{2}
\end{aligned}
$$

Finally, from the condition of $\beta_{k}$ given by (3.4), we get

$$
\left\|x_{k+1}-u\right\|^{2} \leq\left\|x_{k}-u\right\|^{2}-\left(1-v^{2}\right)\left\|x_{k}-y_{k}\right\|^{2} .
$$

Case 2: $f(u)=0$.

From (4.4), we can easily get

$$
\left\|x_{k+1}-u\right\|^{2} \leq\left\|x_{k}-u\right\|^{2}-\left\|x_{k}-y_{k}\right\|^{2}+\beta_{k}^{2}\left\|f\left(x_{k}\right)-f\left(y_{k}\right)\right\|^{2} .
$$

Obviously, (3.4) implies

$$
\beta_{k}^{2}\left\|f\left(x_{k}\right)-f\left(y_{k}\right)\right\|^{2} \leq v^{2}\left\|x_{k}-y_{k}\right\|^{2}, \quad v \in(0,1) .
$$

Thus, (4.1) follows from the combination of (4.10) and (4.11).

Indeed, substituting (3.3) into (3.4), we get

$$
\left(\sigma \rho^{m_{k}}\right)^{2}\left\|f\left(x_{k}\right)-f\left(y_{k}\right)\right\|^{2}+2 M \sigma \rho^{m_{k}}\left\|x_{k}-y_{k}\right\|^{2} \leq v^{2}\left\|x_{k}-y_{k}\right\|^{2} .
$$

Let $m$ be the smallest nonnegative integer, such that

$$
\left(\sigma \rho^{m}\right)^{2} \kappa^{2}+2 M \sigma \rho^{m} \leq v^{2}
$$


where $\kappa$ is the Lipschitz constant of $f$. Noting that $\left\|f\left(x_{k}\right)-f\left(y_{k}\right)\right\| \leq \kappa\left\|x_{k}-y_{k}\right\|$, we assert from (4.12) and (4.13) that $m_{k} \leq m$, which implies

$$
\beta_{k} \geq \sigma \rho^{m},
$$

namely $\inf _{k \geq 0}\left\{\beta_{k}\right\}>0$.

Theorem 4.2 Assume that Conditions 3.1-3.3 hold. Then the two sequences $\left\{x_{k}\right\}_{k=0}^{\infty}$ and $\left\{y_{k}\right\}_{k=0}^{\infty}$ generated by Algorithm 3.4 converge weakly to the same point $z \in \operatorname{SOL}(C, f)$, furthermore

$$
z=\lim _{k \rightarrow \infty} P_{\operatorname{SOL}(C, f)}\left(x_{k}\right)
$$

Proof By Lemma 4.1,

$$
\left\|x_{k+1}-u\right\|^{2} \leq\left\|x_{k}-u\right\|^{2}
$$

for all $k \geq 0$, so there exists

$$
a=\lim _{k \rightarrow \infty}\left\|x_{k}-u\right\|
$$

and the sequence $\left\{x_{k}\right\}_{k=0}^{\infty}$ is bounded. From (4.1), we have

$$
\left\|x_{k}-y_{k}\right\|^{2} \leq \frac{1}{1-v^{2}}\left[\left\|x_{k}-u\right\|^{2}-\left\|x_{k+1}-u\right\|^{2}\right] .
$$

Hence,

$$
x_{k}-y_{k} \rightarrow 0 \quad(k \rightarrow \infty)
$$

In addition,

$$
f\left(x_{k}\right)-f\left(y_{k}\right) \rightarrow 0 \quad(k \rightarrow \infty) .
$$

Using the Cauchy-Schwartz inequality,

$$
\left\|y_{k}\right\|=\left\|y_{k}-x_{k}+x_{k}\right\| \leq\left\|y_{k}-x_{k}\right\|+\left\|x_{k}\right\|,
$$

hence, the sequence $\left\{y_{k}\right\}_{k=0}^{\infty}$ is also bounded.

Let $\omega\left(x_{k}\right)$ be the set of weak limit points of $\left\{x_{k}\right\}_{k=0}^{\infty}$, i.e.,

$$
\omega\left(x_{k}\right)=\left\{z \mid \exists\left\{x_{k_{j}}\right\}_{j=0}^{\infty} \subset\left\{x_{k}\right\}_{k=0}^{\infty} \text { s.t. } x_{k_{j}} \rightarrow z\right\} .
$$

Since the sequence $\left\{x_{k}\right\}_{k=0}^{\infty}$ is bounded, $\omega\left(x_{k}\right) \neq \emptyset$. Taking $z \in \omega\left(x_{k}\right)$ arbitrarily, there exists some subsequence $\left\{x_{k_{j}}\right\}_{j=0}^{\infty}$ of $\left\{x_{k}\right\}_{k=0}^{\infty}$, such that

$$
x_{k_{j}} \rightarrow z \quad(j \rightarrow \infty) .
$$


Equation (4.17) together with (4.16) leads to

$$
y_{k_{j}} \rightarrow z \quad(j \rightarrow \infty)
$$

Due to $y_{k} \in C_{k}$ and the definition of $C_{k}$, we have

$$
c\left(x_{k}\right)-\left\langle c^{\prime}\left(x_{k}\right), x_{k}-y_{k}\right\rangle \leq 0,
$$

then, using the Cauchy-Schwartz inequality again,

$$
c\left(x_{k}\right) \leq\left\|c^{\prime}\left(x_{k}\right)\right\|\left\|x_{k}-y_{k}\right\|
$$

According to (iii) in Condition 3.3, we can deduce that $c^{\prime}(x)$ is bounded on any bounded sets of $H$, so there exists $M^{\prime}>0$ such that $\left\|c^{\prime}\left(x_{k}\right)\right\| \leq M^{\prime}$ for all $k \geq 0$, and then

$$
c\left(x_{k}\right) \leq M^{\prime}\left\|x_{k}-y_{k}\right\| \rightarrow 0 \quad(k \rightarrow \infty) .
$$

According to (ii) in Condition 3.3, we have

$$
c(z) \leq \liminf _{j \rightarrow \infty} c\left(x_{k_{j}}\right) \leq 0 .
$$

Hence, $z \in C$.

Now, we turn to showing $z \in \operatorname{SOL}(C, f)$. Define

$$
T v= \begin{cases}f(v)+N_{C}(v), & \text { if } v \in C, \\ \emptyset, & \text { if } v \notin C,\end{cases}
$$

where $N_{C}(v)$ is defined by (2.5). Obviously, $T$ is a maximal monotone operator.

For arbitrary $(v, w) \in G(T)$, we have

$$
w \in T(v)=f(v)+N_{C}(v)
$$

equivalently,

$$
w-f(v) \in N_{C}(v) .
$$

Setting $y=z$ in (2.5), we get

$$
\langle w-f(v), z-v\rangle \leq 0 \text {. }
$$

On the other hand, by the definition of $y_{k}$ and (2.2), we have

$$
\left\langle x_{k}-\beta_{k} f\left(x_{k}\right)-y_{k}, y_{k}-v\right\rangle \geq 0
$$

or

$$
\left\langle\frac{y_{k}-x_{k}}{\beta_{k}}+f\left(x_{k}\right), v-y_{k}\right\rangle \geq 0
$$


for all $k \geq 0$. Using (4.21) and (4.22), we obtain

$$
\begin{aligned}
&\langle w, v-z\rangle \geq\langle f(v), v-z\rangle \\
& \geq\langle f(v), v-z\rangle-\left\langle\frac{y_{k_{j}}-x_{k_{j}}}{\beta_{k_{j}}}+f\left(x_{k_{j}}\right), v-y_{k_{j}}\right\rangle \\
&=\left\langle f(v), v-y_{k_{j}}+y_{k_{j}}-z\right\rangle-\left\langle\frac{y_{k_{j}}-x_{k_{j}}}{\beta_{k_{j}}}+f\left(x_{k_{j}}\right), v-y_{k_{j}}\right\rangle \\
&=\left\langle f(v), v-y_{k_{j}}\right\rangle+\left\langle f(v), y_{k_{j}}-z\right\rangle \\
&-\left\langle\frac{y_{k_{j}}-x_{k_{j}}}{\beta_{k_{j}}}+f\left(x_{k_{j}}\right), v-y_{k_{j}}\right\rangle \\
&=\left\langle f(v)-f\left(y_{k_{j}}\right), v-y_{k_{j}}\right\rangle+\left\langle f\left(y_{k_{j}}\right)-f\left(x_{k_{j}}\right), v-y_{k_{j}}\right\rangle \\
&-\left\langle\frac{y_{k_{j}}-x_{k_{j}}}{\beta_{k_{j}}}, v-y_{k_{j}}\right\rangle+\left\langle f(v), y_{k_{j}}-z\right\rangle \\
& \geq\left\langle f(v), y_{k_{j}}-z\right\rangle+\left\langle f\left(y_{k_{j}}\right)-f\left(x_{k_{j}}\right), v-y_{k_{j}}\right\rangle \\
&-\left\langle\frac{y_{k_{j}}-x_{k_{j}}}{\beta_{k_{j}}}, v-y_{k_{j}}\right\rangle .
\end{aligned}
$$

By virtue of (4.14), (4.16) and Condition 3.2, taking $j \rightarrow \infty$ in (4.23), we have

$$
\langle w, v-z\rangle \geq 0 .
$$

Since $T$ is a maximal monotone operator, (4.24) means that $0 \in T(z)$ and consequently $z \in T^{-1}(0)=\operatorname{SOL}(C, f)$.

Now we are in a position to verify that $x_{k} \rightarrow z(k \rightarrow \infty)$. In fact, if there exists another subsequence $\left\{x_{k_{i}}\right\}_{i=0}^{\infty}$ of $\left\{x_{k}\right\}_{k=0}^{\infty}$, such that $x_{k_{i}} \rightarrow \bar{z} \in \operatorname{SOL}(C, f)$, but $\bar{z} \neq z$, noting the fact that $\left\{\left\|x_{k}-u\right\|\right\}_{k=0}^{\infty}$ is decreasing for all $u \in \operatorname{SOL}(C, f)$, we obtain by using Lemma 2.3

$$
\begin{aligned}
\lim _{k \rightarrow \infty}\left\|x_{k}-z\right\| & =\lim _{j \rightarrow \infty}\left\|x_{k_{j}}-z\right\|<\lim _{j \rightarrow \infty}\left\|x_{k_{j}}-\bar{z}\right\| \\
& =\lim _{k \rightarrow \infty}\left\|x_{k}-\bar{z}\right\|=\lim _{i \rightarrow \infty}\left\|x_{k_{i}}-\bar{z}\right\| \\
& <\lim _{i \rightarrow \infty}\left\|x_{k_{i}}-z\right\|=\lim _{k \rightarrow \infty}\left\|x_{k}-z\right\| .
\end{aligned}
$$

This is a contradiction, so $\bar{z}=z$. Consequently, we have $x_{k} \rightarrow z(k \rightarrow \infty)$ and $y_{k} \rightarrow z$ $(k \rightarrow \infty)$.

Finally, we show that $z=\lim _{k \rightarrow \infty} P_{\mathrm{SOL}(C, f)}\left(x_{k}\right)$. Put $u_{k}=P_{\mathrm{SOL}\left(C_{f}\right)}\left(x_{k}\right)$, using (2.2) again and $z \in \operatorname{SOL}(C, f)$,

$$
\left\langle x_{k}-u_{k}, u_{k}-z\right\rangle \geq 0 \text {. }
$$


By Lemma 2.4, there exists $u^{*} \in \operatorname{SOL}(C, f)$ such that $u_{k} \rightarrow u^{*}$. Therefore, taking $k \rightarrow \infty$ in (4.26), we have

$$
\left\langle z-u^{*}, u^{*}-z\right\rangle \geq 0
$$

therefore $z=u^{*}$. The proof is complete.

\section{Convergence rate of the modified method}

In this section, we prove the convergence rate of our modified subgradient extragradient method in the ergodic sense. The base of the complexity proof is $([23,24])$

$$
\operatorname{SOL}(C, f)=\bigcap_{u \in C}\{z \in C \mid\langle f(u), u-z\rangle \geq 0\}
$$

In order to prove the convergence rate, now we give the key inequality of our method. Indeed, by an argument very similar to the proof of Lemma 4.1, it is not difficult to get the following result.

Lemma 5.1 Let $\left\{x_{k}\right\}_{k=0}^{\infty}$ and $\left\{y_{k}\right\}_{k=0}^{\infty}$ be the two sequences generated by Algorithm 3.4 and let $\beta_{k}$ be selected as (3.3) and (3.4). Assume that the Conditions 3.1, 3.2 and 3.3 are satisfied. Then, for any $u \in C$, we have

$$
\left\|x_{k+1}-u\right\|^{2} \leq\left\|x_{k}-u\right\|^{2}-\left(1-v^{2}\right)\left\|x_{k}-y_{k}\right\|^{2}+2 \beta_{k}\left\langle f(u), u-y_{k}\right\rangle .
$$

Theorem 5.2 For any integer $n>0$, we have a $z_{n} \in H$, which satisfies $z_{n} \rightarrow z, z \in \operatorname{SOL}(C, f)$ and

$$
\left\langle f(u), z_{n}-u\right\rangle \leq \frac{\left\|x_{0}-u\right\|^{2}}{\Upsilon_{n}}, \quad \forall u \in C,
$$

where

$$
z_{n}=\frac{\sum_{k=0}^{n} 2 \beta_{k} y_{k}}{\Upsilon_{n}} \text { and } \Upsilon_{n}=\sum_{k=0}^{n} 2 \beta_{k}
$$

Proof Using (5.2), we get

$$
2 \beta_{k}\left\langle f(u), y_{k}-u\right\rangle \leq\left\|x_{k}-u\right\|^{2}-\left\|x_{k+1}-u\right\|^{2}
$$

Summing the inequality (5.5) over $k=0,1, \ldots, n$, we get

$$
\left\langle f(u), \sum_{k=0}^{n} 2 \beta_{k} y_{k}-\sum_{k=0}^{n} 2 \beta_{k} u\right\rangle \leq\left\|x_{0}-u\right\|^{2}, \quad \forall u \in C .
$$

From the notation of $\Upsilon_{n}$ and $z_{n}$ in (5.4), we derive

$$
\left\langle f(u), z_{n}-u\right\rangle \leq \frac{\left\|x_{0}-u\right\|^{2}}{\Upsilon_{n}}, \quad \forall u \in C
$$


On the other hand, since $z_{n}$ is a convex combination of $y_{0}, y_{1}, \ldots, y_{n}$, it is easy to see that $z_{n} \rightarrow z \in \operatorname{SOL}(C, f)$ due to the fact that $y_{k} \rightarrow z \in \operatorname{SOL}(C, f)$ proved by Theorem 4.2. The proof is complete.

Let $\beta=\sigma \rho^{m}$. From (4.14), $\beta_{k} \geq \beta$ holds for all $k \geq 0$ and this together with (5.4) leads to

$$
\Upsilon_{n} \geq 2(n+1) \beta,
$$

this means the modified subgradient extragradient method has $O\left(\frac{1}{n}\right)$ convergence rate. In fact, for any bounded subset $D \subset C$ and given accuracy $\epsilon>0$, our algorithm achieves

$$
\left\langle f(u), z_{n}-u\right\rangle \leq \epsilon, \quad \forall u \in D
$$

in at most

$$
n=\left\lceil\frac{m}{2 \beta \epsilon}\right\rceil
$$

iterations, where $z_{n}$ defined in (5.4) and $m=\sup \left\{\left\|x_{0}-u\right\|^{2} \mid u \in D\right\}$.

\section{Results and discussion}

Since the modified subgradient extragradient method proposed in this paper is relaxed and self-adaptive, it is easily implemented. A weak convergence theorem for our algorithm is proved due to the alternating theorem for the solutions of variational inequalities. Our results in this paper effectively improve the existing related results.

\section{Conclusion}

Although the extragradient methods and the subgradient extragradient methods have been widely studied, the existing algorithms all face the problem that the projection operator is hard to calculate. The problem can be solved effectively by using the modified subgradient extragradient method proposed in this paper, since two projections onto the original domain are all replaced with projections onto some half-spaces, which is very easily calculated. Besides, the step size can be selected in some adaptive ways, which means that we have no need to know or to estimate the Lipschitz constant of the operator. Furthermore, we prove that our method has $O\left(\frac{1}{n}\right)$ convergence rate.

Competing interests

The authors declare that they have no competing interests.

Authors' contributions

All authors contributed equally to the writing of this paper. All authors read and approved the final manuscript.

Acknowledgements

This work was supported by the Foundation of Tianjin Key Lab for Advanced Signal Processing (2016 ASP-TJ02).

\section{Publisher's Note}

Springer Nature remains neutral with regard to jurisdictional claims in published maps and institutional affiliations. 


\section{References}

1. Kinderlehrer, D, Stampacchia, G: An Introduction to Variational Inequalities and Their Applications. Society for Industrial and Applied Mathematics, Philadelphia (2000)

2. Korpelevich, GM: An extragradient method for finding saddle points and for other problems. Ėkon. Mat. Metody 12 747-756 (1976)

3. Nadezhkina, N, Takahashi, W: Weak convergence theorem by an extragradient method for nonexpansive mappings and monotone mappings. J. Optim. Theory Appl. 128, 191-201 (2006)

4. Censor, Y, Gibali, A, Reich, S: Extensions of Korpelevich's extragradient method for the variational inequality problem in Euclidean space. Optimization 61, 1119-1132 (2012)

5. Censor, Y, Gibali, A, Reich, S: The subgradient extragradient method for solving variational inequalities in Hilbert space. J. Optim. Theory Appl. 148, 318-335 (2011)

6. Censor, Y, Gibali, A, Reich, S: Strong convergence of subgradient extragradient methods for the variational inequality problem in Hilbert space. Optim. Methods Softw. 26, 827-845 (2011)

7. Yao, YH, Postolache, M, Liou, YC, Yao, ZS: Construction algorithms for a class of monotone variational inequalities. Optim. Lett. 10, 1519-1528 (2016)

8. Yao, YH, Liou, YC, Kang, SM: Approach to common elements of variational inequality problems and fixed point problems via a relaxed extragradient method. Comput. Math. Appl. 59, 3472-3480 (2010)

9. Yao, YH, Noor, MA, Liou, YC, Kang, SM: Iterative algorithms for general multivalued variational inequalities. Abstr. Appl. Anal. 2012, $768272(2012)$

10. Yao, YH, Noor, MA, Liou, YC: Strong convergence of a modified extragradient method to the minimum-norm solution of variational inequalities. Abstr. Appl. Anal. 2012, 817436 (2012)

11. Zegeye, H, Shahzad, N, Yao, YH: Minimum-norm solution of variational inequality and fixed point problem in Banach spaces. Optimization 64, 453-471 (2015)

12. Yao, YH, Shahzad, N: Strong convergence of a proximal point algorithm with general errors. Optim. Lett. 6, 621-628 (2012)

13. He, S, Yang, C: Solving the variational inequality problem defined on intersection of finite level sets. Abstr. Appl. Anal. 2013, 942315 (2013)

14. Fukushima, M: A relaxed projection method for variational inequalities. Math. Program. 35, 58-70 (1986)

15. Takahashi, W: Nonlinear Functional Analysis. Yokohama Publishers, Yokohama (2000)

16. Goebel, K, Reich, S: Uniform Convexity, Hyperbolic Geometry and Non-expansive Mappings. Dekker, New York (1984)

17. Hiriart-Urruty, JB, Lemarchal, C: Fundamentals of Convex Analysis. Springer, Berlin (2001)

18. Rockafellar, RT: On the maximality of sums of nonlinear monotone operators. Trans. Am. Math. Soc. 149, 75-88 (1970)

19. Opial, Z: Weak convergence of the sequence of successive approximations for nonexpansive mappings. Bull. Am. Math. Soc. 73, 591-597 (1967)

20. Takahashi, W, Toyoda, M: Weak convergence theorems for nonexpansive mappings and monotone mappings. J. Optim. Theory Appl. 118, 417-428 (2003)

21. He, S, Xu, HK: Uniqueness of supporting hyperplanes and an alternative to solutions of variational inequalities. J. Glob. Optim. 57, 1375-1384 (2013)

22. Nguyen, $\mathrm{HQ}, \mathrm{Xu}, \mathrm{HK}$ : The supporting hyperplane and an alternative to solutions of variational inequalities. J. Nonlinear Convex Anal. 16, 2323-2331 (2015)

23. Facchinei, F, Pang, JS: Finite-Dimensional Variational Inequalities and Complementarity Problems, vols. I and II. Springer Series in Operations Research. Springer, New York (2003)

24. Cai, $X J, G u, G Y, H e, B S:$ On the $O\left(\frac{1}{t}\right)$ convergence rate of the projection and contraction methods for variational inequalities with Lipschitz continuous monotone operators. Comput. Optim. Appl. 57, 339-363 (2014)

\section{Submit your manuscript to a SpringerOpen ${ }^{\circ}$ journal and benefit from:}

- Convenient online submission

Rigorous peer review

- Immediate publication on acceptance

- Open access: articles freely available online

- High visibility within the field

- Retaining the copyright to your article 